\title{
Stellar and wind properties of massive stars in the central parsec of the Galaxy
}

\author{
Fabrice Martins, Reinhard Genzel, Frank Eisenhauer, Thibaut \\ Paumard, Thomas Ott, Sascha Trippe and Stefan Gillessen \\ MPE, Postfach 1312, D-85741 Garching bei München, Germany \\ email: martins@mpe.mpg.de
}

\begin{abstract}
The stellar and wind properties of the new population of massive stars in the central parsec of the Galaxy are derived through quantitative analysis with atmosphere models.
\end{abstract}

Keywords. stars: atmospheres - stars: early-type - stars: Wolf-Rayet - Galaxy: center

\section{The central cluster}

The central cluster of the Galaxy, which harbors the supermassive black hole SgrA , is a unique environment to study massive stars. In this particularly hostile environment where tidal forces are large, the presence of massive stars tracing recent star formation is puzzling: this is the "paradox of youth" of the Galactic Center.

The first stars observed in the central cluster were evolved massive stars: the so-called He I stars showing strong emission lines in the $K$-band (Najarro et al. 1997). But they were only the tip of the iceberg: in the last years, the development of $3 \mathrm{D}$ spectroscopy has lead to the discovery $\sim 80$ massive stars in the central parsec, including various types of Wolf-Rayet stars (see also van der Hucht 2006) as well as O- and B-type supergiants and dwarfs. This population results from a burst of star formation $\sim 6 \mathrm{Myr}$ ago and is spatially distributed in two counter-rotating disks (Paumard et al. 2006).

\section{Stellar and wind properties}

Quantitative spectroscopic analysis of the Wolf-Rayet population with atmosphere models reveal that these stars are similar to other Galactic WR stars, possibly indicating a common formation process in spite of the peculiar environment of the Galactic Center. Their metallicity appears to be about solar. The new population of early type stars accounts for the $\mathrm{H}$ and He I ionizing fluxes required to ionize the local ISM. This means that contrary to the claim of Lutz (1999), standard stellar evolution does not face any serious problem in the Galactic Center. The He I stars are less chemically evolved than late type WN stars (especially WN8) and we suggest that they may be their precursors. Their mass range is $20-70 \mathrm{M}_{\odot}$, one of them being in a binary system and having a mass of $\sim 50 \mathrm{M}_{\odot}$ (Martins et al. 2006).

\section{References}

van der Hucht, K. A. 2006, A\&A, 458, 453

Lutz, D. 1999, in: P. Cox \& M. F. Kessler (eds.), The Universe as Seen by ISO, ESA SP-427, 623

Martins, F., Trippe, S., Paumard, T., et al. 2006, ApJ (Letters), 649, L103

Najarro, F., Krabbe, A., Genzel, R., et al. 1997, A\&A, 325, 700

Paumard, T., Genzel, R., Martins, F., et al. 2006, ApJ, 643, 1011 